\title{
Training, assessments and examinations in the "new normal" era
}

\author{
Anuja Abayadeera ${ }^{1^{*}}$ \\ Professor in Anaesthesiology, Department of Anaesthesiology and Critical Care, Faculty of Medicine, \\ University of Colombo. Sri Lanka.
}

COVID 19 has devastated the world as a continuing global health crisis and socio-economic disaster. At the time of writing this editorial SARS-CoV-2 is documented to have infected nearly 10 million people and caused close to half a million deaths. ${ }^{1}$ Sri Lanka has a relative success story recording just over 2000 infections and only 11 deaths. $^{2}$

Most countries have suffered weeks of lock down with corresponding economic devastation, the cost in loss of lives and heavy psychological impact being untold.

In this issue of the journal the invited review is on how the United Kingdom National Health Service geared for the expected numbers of infected and the needs for critical care services. ${ }^{3}$

The impact it has had on traditional education, postgraduate training and assessment is immense all over the world. Most anaesthetists in training have had to forego their routine schedules or training modules and move over to manage critically ill COVID 19 patients. Many have themselves got infected in the process. Let's hope and pray that none succumbed during their line of duty.

In Sri Lanka too the designated training schedules could not be adhered to as initially all routine work was curtailed and only emergency work continued. Nevertheless, they needed special training and education in the use of personal protective equipment and identification, resuscitation, and provision of anaesthesia and critical care for COVID 19 infected patients. Due to the limited

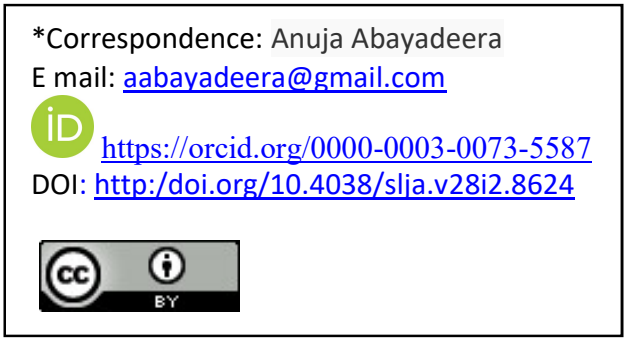

number of critically ill patients in Sri Lanka to date, the exposure to their management was limited. But all trainees were provided access to the relevant guidelines and protocols formulated by the professional colleges together with the Ministry of Health. Formal classroom teaching had to be totally abandoned initially due to 'social distancing' rules and although a few trainers resorted to zoom conferencing, this was quite limited.

As we move on to the so called 'new normal' era we must adapt to a world where life and work as we knew it will no longer be possible. We need to plan and organize both short term and long-term measures to ensure that professional development and quality patient care will continue uninterrupted.

\section{Training}

It is important to think outside the box for any novel methods of training that we could embark on. The core 'hands on' training for an anaesthetist is unlikely to undergo any changes, although an everincreasing stress is being laid on simulation training with mannequins and reality-based scenarios with high tech equipment. The general rule at present is that the scheduled rotations would continue, and the missed training opportunities would be allocated at the end of the training course prior to the final examinations. The Royal College of Anaesthetists United Kingdom ${ }^{4}$ and the Australian and New Zealand College of Anaesthetists ${ }^{5}$ have spelt out the training requirements that need to be fulfilled.

Formal classroom teaching can continue with reasonable social distancing. Presentations, journal clubs, case discussions and question and answer sessions utilizing online conferencing platforms may soon be the new norm. Trainees are also encouraged to attend international webinars conducted by many organizations which would be of great benefit. These are educational resources that all trainees should use regularly for their advancement. The Sri Lanka Medical Association has used local resources to conduct webinars and 
paved the way forward for us anaesthetists to think of conducting training workshops and demonstrations using a conferencing portal.

\section{Assessments}

In service assessments are a dilemma at present as reviews of progression of training have not been completed for many batches of current trainees. The Board of Study in Anaesthesiology is in the process of trying to organize virtual assessments once relevant documentation has been submitted to the Postgraduate Institute of Medicine. The initial assessments would be conducted for the Senior Registrars coming up for Board Certification and for final review of progression. The documentation would have to be provided in an organized manner to ease the conduct of the assessment. The Royal College of Anaesthetists is planning the Annual Review of Progression (ARCP) in absentia with assessment of the submitted documents and provision of an outcome assessment. ${ }^{4}$ Would virtual assessments be the new normal? The formalized presentation of documents by the trainees are essential at present but maybe virtual in the future!

\section{Examinations}

Our most serious concern is the conduct of the selection and final examinations in Anaesthesiology. Postponement of examinations have occurred in most countries, but the goal is to return to normal schedules as soon as feasible. In this context, we plan to hold the examinations as scheduled to get back to near normalcy. The final examination entails an important clinical component with patients who may have to be screened for infection risk prior to exposure to candidates which would be a challenge to the organizers. Undergraduate examinations conducted by the universities currently would be a useful guide to determine the feasibility of clinical examinations.

All postgraduate examinations will be handicapped by the absence of external overseas examiners this year due to quarantine regulations discouraging travel. The uncertainty of the dates of the examinations undoubtedly would have added to the stress of the candidates. The MD Anaesthesiology final examination is conducted with physicians as examiners for the clinical component and therefore we do have some 'external' input. Examinations this year for anaesthesiology would be quality controlled by overseas appointed examiners from the Royal College of Anaesthetists, London via video conferencing. This exercise would be challenging to both local and overseas examiners and to the observed candidates. We need to consider the time difference with the United Kingdom in scheduling the examination components that require quality control and ensure that technological mishaps do not occur. I hope the resilience of our examiners and the candidates would see us through this difficult and challenging task. Robust technology is also a factor for success of this attempt. The Royal College of Anaesthetists, UK is planning online remotely invigilated written examinations and are trialing virtual structured oral examinations ${ }^{4}$, which may well be a role model for us in the future.

Their dilemma is also the conduct of the structured clinical examinations which may well be need the presence of patients or individuals, examiners and the candidates in an environment. It is perhaps time to reflect on whether such examinations are absolutely essential. Even in the past some doubt has been cast on the validity of such subjective assessment in a short period of time. An in-depth study comparing the results of such examinations with the progress reports of trainers throughout rotational training program would be worthwhile. Do we need to place such absolute importance on an end of training "high stakes" examination? If highly supervised training and adequate assessment has been sufficiently built into an intensive 3 year program, what more could another half hour of testing add? It is not easy to break with tradition, but a standardized, reliable, training program based on transparency and equity, should ensure consistency and quality control.

If, however some form of end of training examination is deemed essential it may be worthwhile considering other options. In this context it is noteworthy that the Imperial College London claimed to have has the first ever successful online "open book" examination for 280 final year medical students in March this year. This month the University of Colombo conducted the MBBS Final clinical examination in Surgery via zoom conferencing for a student in Bhutan.

As clinicians and educationists, we need to rise to 
unprecedented challenges with innovative and dynamic solutions where we may have to give up many of our preconceived notions of traditional training and assessment and harness all the hightech resources available so that we may forge ahead.

\section{References}

1. https://www.worldometers.info/coronavirus/

2. https://www.epid.gov.lk/web/images/pdf/corona_vi rus_report

3. Warnapura C. Sri Lankan Journal of Anaesthesiology. 2020; 28(2):62-67

4. https://rcoa.ac.uk/training-careers

5. http://www.anzca.edu.au/training 